Failure of these arterial twigs to fuse result in the twig-like or unfused MCA.

Methods A retrospective single case report of twig-like MCA with literature review. The patient established care at our institution in 6/2018 and still follows with us.

Results A 55-year-old woman with a past medical history of hyperlipidemia and tobacco use presented in the outpatient setting with an electrical-like sensation over her right neck. She denied focal weakness, speech difficulty, visual problems or numbness. There was no history of trauma or neck manipulation. She has a family history of stroke and aneurysm. She was evaluated by magnetic resonance angiography (MRA) of the head which showed a small right internal carotid artery (ICA) and high-grade stenosis of the right ICA and right MCA. Computed tomography (CT) angiography demonstrated small caliber of the entire right ICA and critical stenosis versus occlusion of the right ICA and MCA with distal reconstitution compatible with a moyamoya pattern with lenticulostriate collateralization. CT brain perfusion demonstrated a significant reduction in cerebral blood flow of the entire right MCA territory without a decrease in cerebral blood volume. Cerebral angiography revealed a right twig-like MCA with contralateral ICA cavernous segment aneurysm. The patient has been treated conservatively with aspirin and atorvastatin.

The figure 1 illustrates the right internal common carotid injection with $3 \mathrm{D}$ rotational angiography reconstruction image (A) and AP subtraction image (B). The right M1 segment of the middle cerebral artery has a plexiform arterial network that is consistent with an unfused middle cerebral artery. There are no moyamoya vessels in the basal region and no occlusion in the terminus segment of the internal carotid artery. The twig-like artery can be traceable to the distal M1 or proximal M2 segment. There is no intracranial atherosclerotic change.

Conclusion Twig-like or unfused MCA are rare vascular anomalies that may be mistaken for moyamoya disease. The detailed trans-femoral cerebral angiogram interpretation of the twig-like MCA depicts distinctive differences from the findings of moyamoya disease.

Disclosures A. Schnure: 4; C; General Electric, CYTRX, Portola Pharmaceuticals. A. Sweidan: None. I. Yuki: None. S. Suzuki: None.

\section{E-061 TRANSVERSE VENOUS SINUS STENOSIS AS A TREATABLE CAUSE OF IDIOPATHIC INTRACRANIAL HYPERTENSION: AN UNDER-RECOGNIZED ENTITY}

P Ramakrishnan*, C Berry, S Leinfelder, W Leesch, J Sanderson. Neurovascular Specialists, Riverside Regional Medical Center, Newport News, VA

\subsection{6/neurintsurg-2019-SNIS. 136}

Introduction and purpose Interventional treatment for transverse sinus stenosis with venous sinus stenting is emerging as a viable, and minimally invasive treatment for medically refractory idiopathic intracranial hypertension (IIH). However, this therapeutic intervention remains under-recognized and often accompanied by limited diagnostic evaluation and neurointerventional referral. We present the commonly employed neuroimaging paradigm in the South Eastern Virginia population with IIH, and seek to highlight areas of improvement to better stratify patients based on disease mechanism, incorporating neuroanatomic and pathophysiologic considerations.

Materials and methods We conducted a retrospective chart review within our health system from January 2018 to September 2018, extracting clinical data from 70 cases. From this data, 59 were categorized as confirmed $\mathrm{IIH}$, with 11 presently undergoing workup. The following variables were analyzed in those with confirmed IIH: patient demographics, presenting symptoms, papilledema, and neuroimaging.

Results Fifty-seven female and two male patients with confirmed IIH ( $\mathrm{n}=59)$ with a mean age of 38 and BMI of 39.2 exhibited headache (98\%), vision changes (80\%), pulsesynchronous tinnitus (48\%), and papilledema (64\%) at disease onset. Median cerebrospinal fluid (CSF) opening pressure was $36 \mathrm{~cm} \mathrm{H} 2 \mathrm{O}$. Extent of diagnostic evaluation was assessed and retrospective review of MR venography (MRV) conducted. Twenty-eight (47.4\%) patients in this cohort underwent MRV imaging, amongst whom 20 (71.4\%) exhibited asymmetric venous sinuses or evidence of venous sinus stenosis. Two of the 20 patients (10\%) were referred for neurointerventional evaluation based on suspected transverse sinus stenosis as the mechanism for refractory IIH. Both patients underwent successful transvenous sinus stenting with resolution of transvenous sinus pressure gradient, and clinical symptoms.

Conclusion In this retrospective cohort of IIH, fewer than half of these patients underwent MRV to evaluate for transverse
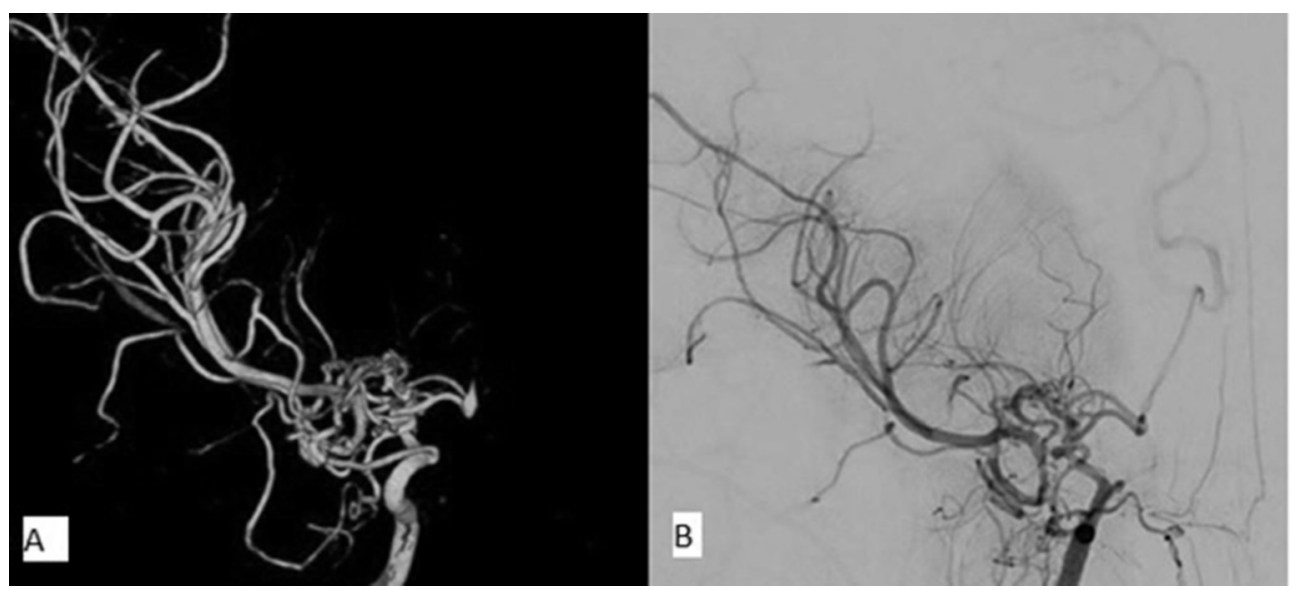

Abstract E-060 Figure 1 
sinus stenosis as a possible mechanism. However, when evaluated with an MRV, over $2 / 3$ of these patients demonstrated asymmetric venous sinus or frank stenosis. The referral of such patients to neurointervention was rare. Our study highlights the overall under-recognition of transverse venous sinus asymmetry/stenosis as a treatable entity and stresses the importance of obtaining an MRV and referring patients for neurointerventional evaluation.

Disclosures P. Ramakrishnan: 3; C; Cerenovus. C. Berry: None. S. Leinfelder: None. W. Leesch: 3; C; Penumbra Inc. 4; C; Cerebrotech Inc. 6; C; Stryker, Medtronic, Terumo. J. Sanderson: None.

\section{E-062 SUBDURAL CONTRAST EFFUSION DURING ENDOVASCULAR THERAPY}

${ }^{1} \mathrm{R}$ Dahl ${ }^{*},{ }^{2} \mathrm{~V}$ Eskesen, ${ }^{1} \mathrm{G}$ Benndorf. 'Department of Radiology, University Hospital Rigshospitalet, Copenhagen, Denmark; ${ }^{2}$ Department of Neurosurgery, University Hospital Rigshospitalet, Copenhagen, Denmark

\subsection{6/neurintsurg-2019-SNIS.137}

Background Accumulation of contrast medium in the subdural space after diagnostic intraarterial and intravenous contrast administrations is a rare observation. The authors report the case of a subdural contrast effusion (SCE) mimicking an acute subdural hematoma ( $\mathrm{SDH}$ ) presenting during embolization of an intracranial dural arteriovenous fistula (DAVF).

Clinical history A 52-year-old woman was admitted to our department with a four months history of left-sided numbness of the upper lip, cheek, tongue and forehead. Digital subtraction angiography showed a right tentorial DAVF with a venous ectasia. The DAVF was mainly supplied by the right middle meningeal artery, occipital artery and the artery of Bernasconi-Cassinari and drained into the Galenic venous system. Procedure The patient underwent endovascular treatment with triaxial catheterization of the right occipital artery. Transarterial embolization using PHIL was performed and monitored by control runs made with an intermediate (distal access) catheter. After several injections, increased accumulation of contrast medium along the cerebellar tentorium and the walls of both transverse sinuses was noted. An immediately performed Dyna CT showed extensive contrast medium in the subdural space of the posterior cranial fossa and foramen magnum suspicious for an acute SDH. The catheters were removed, and the procedure was terminated. The patient woke up presenting no new symptoms and had an uneventful postoperative course. A 24-hour follow-up Dyna CT was completely normal suggesting the diagnosis of an asymptomatic SCE. We believe that repeat high pressure contrast injections via a large bore intermediate catheter into the territory of a (even partly) occluded DAVF may have induced leakage of contrast medium into the extravascular subdural space thereby causing a SCE.

Summary In conclusion we present the unique case of an asymptomatic SCE, which developed during transarterial embolization of a DAVF. SCE can occur during endovascular therapy and may mimic an acute SDH. Differentiation between the two by computed tomography or Dyna CT and early neurological examination can be crucial for patient management.
Disclosures R. Dahl: None. V. Eskesen: None. G. Benndorf: None.

\section{E-063 RECURRENT SYNCOPE CAUSED BY A DURAL ARTERIOVENOUS FISTULA: A CASE REPORT}

D Sheinberg*, E Luther, D McCarthy, R Starke. University of Miami Miller School of Medicine, Miami, FL

\subsection{6/neurintsurg-2019-SNIS.138}

Introduction Dural arteriovenous fistulas (DAVFs) are pathologic vascular connections that shunt dural arterial flow directly to dural venous drainage. DAVFs constitute 10-15\% of all intracranial arteriovenous malformations and are most commonly located in transverse, sigmoid, and cavernous sinuses. Symptoms from DAVFs vary based on lesion location and correlate with their respective patterns of venous drainage. Common presenting symptoms include pulsatile tinnitus, chronic headache, vision difficulties, cranial nerve abnormalities, cerebellar symptoms, focal neurologic deficits, and seizures. Only a few isolated case reports describe syncope on presentation, all in combination with other symptoms. In this article, we report a rare case of DAVF causing recurrent syncope.

Methods A 29 year-old female presented with a 9-year history of progressive syncopal episodes exacerbated by positional changes, strenuous activity and emotional stressors.

Results The patient was referred to us after symptoms of dizziness and syncope persisted despite treatment by multiple cardiologists, endocrinologists, and psychiatrists. Symptoms occurred upon wakening and lasted for 2-3 hours before she was able to regain functionality. Furthermore, she found that her symptoms would remit if she was to lay in the Trendelenburg position immediately after symptom onset. Physical exam revealed no abnormalities. MRI of the brain showed no irregularities. MRA revealed abnormal serpiginous structures in the left jugular foramen which communicated with the ascending pharyngeal branch of the left external carotid artery. Cerebral angiogram disclosed a left jugular foramen dural AVF (Borden/Cognard type 1) supplied by the left ascending pharyngeal and left occipital arteries. There was no retrograde flow or cortical venous reflux. The DAVF was successfully managed by progressive endovascular embolization with coils and Onyx 34 via transvenous route. The final cerebral angiogram demonstrated complete obliteration of the fistula. On clinical follow-up evaluation, the patient had no further episodes of dizziness or syncope.

Conclusion We present an atypical case of DAVF in a patient presenting with recurrent syncope. Only 3 cases of DAVF causing syncope have been reported, all in combination with other neurologic symptoms. In comparison, we report a unique case of DAVF presenting solely with recurrent syncope, a previously undocumented finding in the literature. Our case adds to other reports of nonspecific DAVF presentations and highlights the importance of considering this etiology.

Disclosures D. Sheinberg: None. E. Luther: None. D. McCarthy: None. R. Starke: None. 